\title{
Postoperative Neurologic Deficits in a Veteran With Recent COVID-19
}

\author{
Brian Tolly, MD; Asish Abraham, MD; Malik Ghannam, MBBCh; Jamie Starks, MD; and Liviu Poliac, MD
}

\begin{abstract}
Anesthesia providers should be aware of COVID-19 sensitive stroke code practices and maintain heightened vigilance for the need to implement perioperative stroke mitigation strategies.
\end{abstract}

\begin{abstract}
Brian Tolly is Assistant Professor, Department of Anesthesiology; Asish Abraham is a Resident, Department of Anesthesiology;

Malik Ghannam is a Resident, Department of Neurology; and Jamie Starks is an Assistant Professor, Department of Neurology; all at the University of Minnesota School of Medicine. Liviu Poliac and Brian Tolly are Staff Anesthesiologists and Jamie Starks is a Neurologist in the Geriatric Research Education \& Clinical Center, at the Minneapolis Veterans Affairs Health Care System in Minnesota.

Correspondence: Brian Tolly

(tolly.brian@gmail.com)
\end{abstract}

Fed Pract. 2021;38(3):140-144. doi:10.12788/fp.0094
$\mathrm{T}$ he risk of perioperative stroke in noncardiac, nonneurologic, nonvascular surgery ranges from 0.1 to $1.9 \%$ and is associated with increased mortality. ${ }^{1,2}$ Stroke mechanisms include both ischemia (large and small vessel occlusion, cardioembolism, anemic-tissue hypoxia, cerebral hypoperfusion) and hemorrhage. ${ }^{1}$ Risk factors for perioperative stroke include prior cerebral vascular accident (CVA), hypertension, aged $>62$ years, acute renal insufficiency, dialysis, and recent myocardial infarction (MI). ${ }^{2}$

\section{INTRODUCTION}

COVID-19 was declared a pandemic by the World Health Organization in March $2020 .{ }^{3}$ COVID-19 has certainly affected the veteran population; between February and May 2020, more than 60,000 veterans were tested for COVID-19 with a positive rate of about $9 \% .{ }^{4}$ While primarily affecting the respiratory system, there are increasing reports of COVID-19 neurologic manifestations: headache, hypogeusia, hyposomia, seizure, encephalitis, and acute stroke. ${ }^{5}$ In an early case series from Wuhan, China, $36 \%$ of 214 patients with COVID-19 reported neurologic complications, and acute CVAs were more common in patients with severe (compared to milder) viral disease presentations ( $5.7 \%$ vs $0.8 \%$ ). ${ }^{6}$ Large vessel stroke was a presenting feature in another report of 5 patients aged $<50$ years. ${ }^{7}$

The mechanism of ischemic stroke in the setting of COVID-19 is unclear. ${ }^{8}$ Indeed, stroke and COVID-19 share similar risk factors (eg, hypertension, diabetes mellitus [DM], older age), and immobile critically ill patients may already be prone to developing stroke. ${ }^{5,9}$ However, COVID-19 is associated with arterial and venous thromboembolism, elevated D-dimer and fibrinogen levels, and antiphospholipid antibody production. This prothrombotic state may be linked to cytokine-induced endothelial damage, mononuclear cell activation, tissue factor expression, and ultimately thrombin propagation and platelet activation. ${ }^{8}$

The rates of perioperative stroke may change as more patients with COVID-19 present for surgery, and the anesthesiology care team must prioritize mitigation efforts in high-risk patients, including veterans. Reducing the elevated stroke burden within the US Department of Veterans Affairs (VA) Veterans Health Administration (VHA) is a public health priority. ${ }^{10}$ We present the case of a veteran with prior CVA and recent positive COVID-19 testing who experienced transient weakness and dysarthria following plastic surgery. The patient discussed provided written Health Insurance Portability and Accountability Act consent for publication of this report.

\section{CASE PRESENTATION}

A 75-year-old male veteran presented to the Minneapolis VA Medical Center in Minnesota with chronic left foot ulceration necessitating debridement and flap coverage. His medical history was significant for hypertension, type $2 \mathrm{DM}$, anemia of chronic disease, and coronary artery disease (left ventricular ejection fraction, 50\%). Additionally, he had prior ischemic strokes in the oculomotor nucleus (in 2004 with internuclear ophthalmoplegia) and left ventral medulla (in 2019 with right hemiparesis). During his 2019 poststroke rehabilitation, he was diagnosed with mild 
neurocognitive deficit not attributable to his strokes. The patient's medications included amlodipine, lisinopril, atorvastatin, clopidogrel (lifelong for secondary stroke prevention), metformin, and glipizide. The debridement procedure was initially delayed 3 weeks due to positive routine preoperative COVID-19 nasopharyngeal testing, though he reported no respiratory symptoms or fever. During the delay, the primary team prescribed daily oral rivaroxaban for thrombosis prophylaxis in addition to clopidogrel. One week prior to surgery, his repeat COVID19 test was negative and prophylactic anticoagulation stopped.

On the day of surgery, the patient was hemodynamically stable: heart rate 86 beats/min, blood pressure 167/93 mm Hg (baseline 120-150 mm Hg systolic pressure), respiratory rate 16 breaths/min, oxygen saturation $99 \%$ without supplemental oxygen, temperature $97.1^{\circ} \mathrm{F}$. He received amlodipine and clopidogrel, but not lisinopril, that morning. No focal neurologic deficits were appreciated on preoperative examination, and resolution of symptoms related to the 2 prior MIs was confirmed. Preoperative glucose was $163 \mathrm{mg} / \mathrm{dL}$. Femoral and sciatic peripheral nerve blocks were done for postoperative analgesia. A preinduction arterial line was placed and $2 \mathrm{mg}$ of midazolam was administered for anxiolysis. Induction of general anesthesia with oral endotracheal intubation proceeded uneventfully; he was positioned prone.

Given his stroke risk factors, mean arterial pressure was maintained $>70 \mathrm{~mm} \mathrm{Hg}$ for the duration of surgery. No vasoactive infusions were necessary and no $\beta$-blocking agents were administered. Insulin infusion was required; the maximum-recorded glucose was $219 \mathrm{mg} / \mathrm{dL}$. Arterial blood gas samples were routinely drawn; acid-base balance was well maintained, $\mathrm{PaO}_{2}$ was $>185 \mathrm{~mm} \mathrm{Hg}$, and $\mathrm{PaCO}_{2}$ ranged from 29.4 to $38.5 \mathrm{~mm} \mathrm{Hg}$. The patient received 2 units of packed red blood cells for nadir hemoglobin of $7.5 \mathrm{mg} / \mathrm{dL}$. At surgery end, we fully reversed neuromuscular blockade with suggamadex. The patient was returned to a supine position and extubated uneventfully after demonstrating the ability to follow commands.

During postanesthesia care unit (PACU) handoff, the patient exhibited acute speech impairment. He was able to state his name on repetition but seemed confused and sedated. Prompt formal neurology evaluation (stroke code) was sought. Initial National Institutes of Health (NIH) stroke scale score was 8 ( 1 for level of consciousness, 1 for minor right facial droop, 1 for right arm drift, 3 for right leg with no effort against gravity, 1 for right partial sensory loss, and 1 for mild dysarthria). The patient was oriented only to self. Other findings included mild right facial droop and dysarthria. On a 5-point strength scale, he scored 4 for the right deltoid, biceps, triceps, wrist extensors, right knee flexion, right dorsiflexion, and plantarflexion, 2 for right hip flexion, and $\geq 4$ for right knee extension. Positive sensory findings were notable for decreased pin prick sensation on the right limbs.

We obtained emergent head computed tomography (CT) that was negative for acute abnormalities; CT angiography was negative for large vessel occlusion or clinically significant stenosis (Figure). On returning to the PACU from the CT scanner, the patient regained symmetric strength in both arms, right leg was antigravity, and his speech had normalized. Prior 
to PACU discharge 2 hours later, the patient was back to his prehospitalization neurologic function and NIH stroke scale was 0 . Given this rapid clinical resolution, no acute stroke interventions were done, though permissive hypertension was recommended by the neurologist during PACU recovery.

The neurology team concluded that the patient's symptoms were likely secondary to recrudescence of previous stroke symptoms in the setting of brief postoperative delirium (POD). However, we could not exclude transient ischemic attack or new cardioembolism, therefore patient was started on dual antiplatelet therapy for 3 weeks. Unfortunately, elective confirmatory magnetic resonance imaging (MRI) was not sought to confirm new ischemic changes due hospital COVID-19 restrictions on nonessential scanning. Neurology did not recommend carotid duplex ultrasound given patent vasculature on the head and neck CT angiography. Finally, the patient had undergone surface echocardiography 3 weeks prior to surgery that showed a left ventricular ejection fraction of 50\% without significant valvular abnormalities, thrombus, or interatrial shunting, so repeated study was deferred.

Formal neurology consultation did not extend beyond postoperative day 1 . One month after surgery, the anesthesiology team visited the patient during inpatient rehabilitation; he had not developed further focal neurologic symptoms or delirium. His strength was equal bilaterally and no speech deficits were noted. Unfortunately, the patient was readmitted to the hospital for continued foot wound drainage 2 months postoperatively, though no focal neurologic deficits were documented on his medical admission history and physical. No long term sequalae of his COVID-19 infection have been suspected.

\section{DISCUSSION}

We report a veteran with prior stroke and COVID-19 who experienced postoperative speech and motor deficit despite deliberate risk factor mitigation. This case calls for increased vigilance by anesthesia providers to employ proper perioperative stroke management and anticoagulation strategies, and to be prepared for prompt intervention with
COVID-19-sensitive practices should the need for advanced airway management or thrombectomy arises.

The exact etiology of the postoperative neurologic deficit in our patient is unknown. The most likely possibility is that this represents poststroke recrudescence (PSR), knowing he had a previous left medullary infarct that presented similarly. ${ }^{11}$ PSR is a phenomenon in which prior stroke symptoms recur acutely and transiently in the setting of physiologic stressors-also known as locus minoris resistantiae. ${ }^{12}$ Triggers include $\gamma$ aminobutyric acid (GABA) mediating anesthetic agents such as midazolam, opioids (eg, fentanyl or hydromorphone), infection, or relative cerebral hypoperfusion. . $^{11,13,14}$ The focality of our patient's presentation favors PSR in the context of brief POD; of note, these entities share similar risk factors. ${ }^{15}$ Our patient did indeed receive low-dose preoperative midazolam in the context of mild preoperative neurocognitive deficit, which may have predisposed him to POD.

Though less likely, our patient's presentation could have been explained by a new cerebrovascular event-transient ischemic attack vs new MI. Speech and right-sided motor/sensory deficits can localize to the left middle cerebral artery or small penetrating arteries of the left brainstem or deep white matter. MRI was not performed to exclude this possibility due to hospital-wide COVID-19 precautions minimizing nonessential MRIs unlikely to change clinical management. We speculate, however, that due to recent SARS-CoV-2 infection, our patient may have been at higher risk for cerebrovascular events due to subclinical endothelial damage and/or microclot in predisposed neurovasculature. Though our patient had interval COVID-19 negative tests, the timeframe of coronavirus procoagulant effects is unknown. ${ }^{16}$

There are well-established guidelines for perioperative stroke management published by the Society for Neuroscience in Anesthesiology and Critical Care (SNACC). ${ }^{17}$ This case exemplifies many recommendations including tight hemodynamic and glucose control, optimized oxygen delivery, avoidance of intraoperative $\beta$ blockade, and prompt neurologic consultation. Additionally, special precaution was taken 
to ensure continuation of antiplatelet therapy on the day of surgery; in light of COVID-19 prothrombosis risk we considered this essential. Low-dose enoxaparin was also instituted on postoperative day 1. Prophylactic anticoagulation with low molecular weight heparin (LMWH) is recommended for hospitalized COVID-19positive patients, though perioperatively, this must be weighed against hemorrhagic stroke transformation and surgical bleeding. ${ }^{8,16}$ Interestingly, the benefit of LMWH may partly relate to its anti-inflammatory effects, of which higher levels are observed in COVID-19.16,18

Though substantial health care provider energy and hospital resource utilization is presently focused on controlling the COVID-19 pandemic, the importance of appropriate stroke code processes must not be neglected. Recently, SNACC released anesthetic guidelines for endovascular ischemic stroke management that reflect COVID-19 precautions; highlights include personal protective equipment (PPE) utilization, risk-benefit analysis of general anesthesia (with early decision to intubate) vs sedation techniques for thrombectomy, and airway management strategies to minimize aerosolization exposure. ${ }^{19}$ Finally, negative pressure rooms relative to PACU and operating room locations need to be known and marked, as well as the necessary airway equipment and PPE to transfer patients safely to and from angiography suites.

\section{CONCLUSIONS}

We discuss a surgical patient with prior SARS-CoV-2 infection at elevated stroke risk that experienced recurrence of neurologic deficits postoperatively. This case informs anesthesia providers of the broad differential diagnosis for focal neurological deficits to include PSR and the possible contribution of COVID-19 to elevated acute stroke risk. Perioperative physicians, including VHA practitioners, with knowledge of current COVID-19 practices are primed to coordinate multidisciplinary efforts during stroke codes and ensuring appropriate anticoagulation.

\section{Acknowledgments}

The authors would like to thank perioperative care teams across the world caring for COVID-19 patients safely.
Author disclosures

The authors report no actual or potential conflicts of interest with regard to this article.

\section{Disclaimer}

The opinions expressed herein are those of the authors and do not necessarily reflect those of Federal Practitioner, Frontline Medical Communications Inc., the US Government, or any of its agencies. This article may discuss unlabeled or investigational use of certain drugs. Please review the complete prescribing information for specific drugs or drug combinations-including indications, contraindications, warnings, and adverse effects-before administering pharmacologic therapy to patients.

\section{References}

1. Vlisides P, Mashour GA. Perioperative stroke. Can J Anaesth. 2016;63(2):193-204. doi:10.1007/s12630-015-0494-9

2. Mashour GA, Shanks AM, Kheterpal S. Perioperative stroke and associated mortality after noncardiac, nonneurologic surgery. Anesthesiology. 2011;114(6):1289-1296. doi:10.1097/ALN.0b013e318216e7f4

3. Cucinotta D, Vanelli M. WHO Declares COVID-19 a Pandemic. Acta Biomed. 2020;91(1):157-160. Published 2020 Mar 19. doi:10.23750/abm.v91i1.9397

4. Rentsch CT, Kidwai-Khan F, Tate JP, et al. Covid19 by Race and Ethnicity: A National Cohort Study of 6 Million United States Veterans. Preprint. medRxiv. 2020;2020.05.12.20099135. Published 2020 May 18. doi:10.1101/2020.05.12.20099135

5. Montalvan V, Lee J, Bueso T, De Toledo J, Rivas K. Neurological manifestations of COVID-19 and other coronavirus infections: A systematic review. Clin Neurol Neurosurg. 2020;194:105921. doi:10.1016/j.clineuro.2020.105921

6. Mao L, Jin H, Wang M, et al. Neurologic Manifestations of Hospitalized Patients With Coronavirus Disease 2019 in Wuhan, China. JAMA Neurol. 2020;77(6):683-690. doi:10.1001/jamaneurol.2020.1127

7. Oxley TJ, Mocco J, Majidi S, et al. Large-Vessel Stroke as a Presenting Feature of Covid-19 in the Young. $N$ Engl $J$ Med. 2020;382(20):e60. doi:10.1056/NEJMc2009787

8. Beyrouti R, Adams ME, Benjamin L, et al. Characteristics of ischaemic stroke associated with COVID-19. $J$ Neurol Neurosurg Psychiatry. 2020;91(8):889-891. doi:10.1136/jnnp-2020-323586

9. Needham EJ, Chou SH, Coles AJ, Menon DK. Neurological Implications of COVID-19 Infections. Neurocrit Care. 2020;32(3):667-671. doi:10.1007/s12028-020-00978-4

10. Lich KH, Tian Y, Beadles CA, et al. Strategic planning to reduce the burden of stroke among veterans: using simulation modeling to inform decision making. Stroke. 2014;45(7):2078-2084. doi:10.1161/STROKEAHA.114.004694

11. Topcuoglu MA, Saka E, Silverman SB, Schwamm LH, Singhal AB. Recrudescence of Deficits After Stroke: Clinical and Imaging Phenotype, Triggers, and Risk Factors. JAMA Neurol. 2017;74(9):1048-1055. doi:10.1001/jamaneurol.2017.1668

12. Jun-O'connell AH, Henninger N, Moonis M, Silver B, lonete C, Goddeau RP. Recrudescence of old stroke deficits among transient neurological attacks. Neurohospitalist. 2019;9(4):183-189. doi:10.1177/1941874419829288

13. Karnik HS, Jain RA. Anesthesia for patients with prior stroke. J Neuroanaesthesiology Crit Care. 2018;5(3):150157. doi:10.1055/s-0038-1673549

14. Minhas JS, Rook W, Panerai RB, et al. Pathophysiologi$\mathrm{cal}$ and clinical considerations in the perioperative care of patients with a previous ischaemic stroke: a multidisciplinary narrative review. Br J Anaesth. 2020;124(2):183196. doi:10.1016/j.bja.2019.10.021

15. Aldecoa C, Bettelli G, Bilotta F, et al. European Society of Anaesthesiology evidence-based and consensus-based guideline on postoperative delirium [published correction appears in Eur J Anaesthesiol. 2018 Sep;35(9):718- 
719]. Eur J Anaesthesiol. 2017;34(4):192-214. doi:10.1097/EJA.0000000000000594

16. Thachil J, Tang N, Gando S, et al. ISTH interim guidance on recognition and management of coagulopathy in COVID-19. J Thromb Haemost. 2020;18(5):1023-1026. doi:10.1111/jth.14810

17. Mashour GA, Moore LE, Lele AV, Robicsek SA, Gelb AW. Perioperative care of patients at high risk for stroke during or after non-cardiac, non-neurologic surgery: consensus statement from the Society for Neuroscience in Anesthesiology and Critical

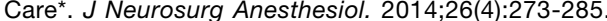
doi:10.1097/ana.0000000000000087

18. Ghannam M, Alshaer Q, Al-Chalabi M, Zakarna L, Robertson J, Manousakis G. Neurologi- cal involvement of coronavirus disease 2019: a systematic review. J Neurol. 2020;267(11):3135-3153. doi:10.1007/s00415-020-09990-2

19. Sharma D, Rasmussen M, Han R, et al. Anesthetic Management of Endovascular Treatment of Acute Ischemic Stroke During COVID-19 Pandemic: Consensus Statement From Society for Neuroscience in Anesthesiology \& Critical Care (SNACC): Endorsed by Society of Vascular \& Interventional Neurology (SVIN), Society of Neurolnterventional Surgery (SNIS), Neurocritical Care Society (NCS), European Society of Minimally Invasive Neurological Therapy (ESMINT) and American Association of Neurological Surgeons (AANS) and Congress of Neurological Surgeons (CNS) Cerebrovascular Section. J Neurosurg Anesthesiol. 2020;32(3):193-201. doi:10.1097/ANA.0000000000000688

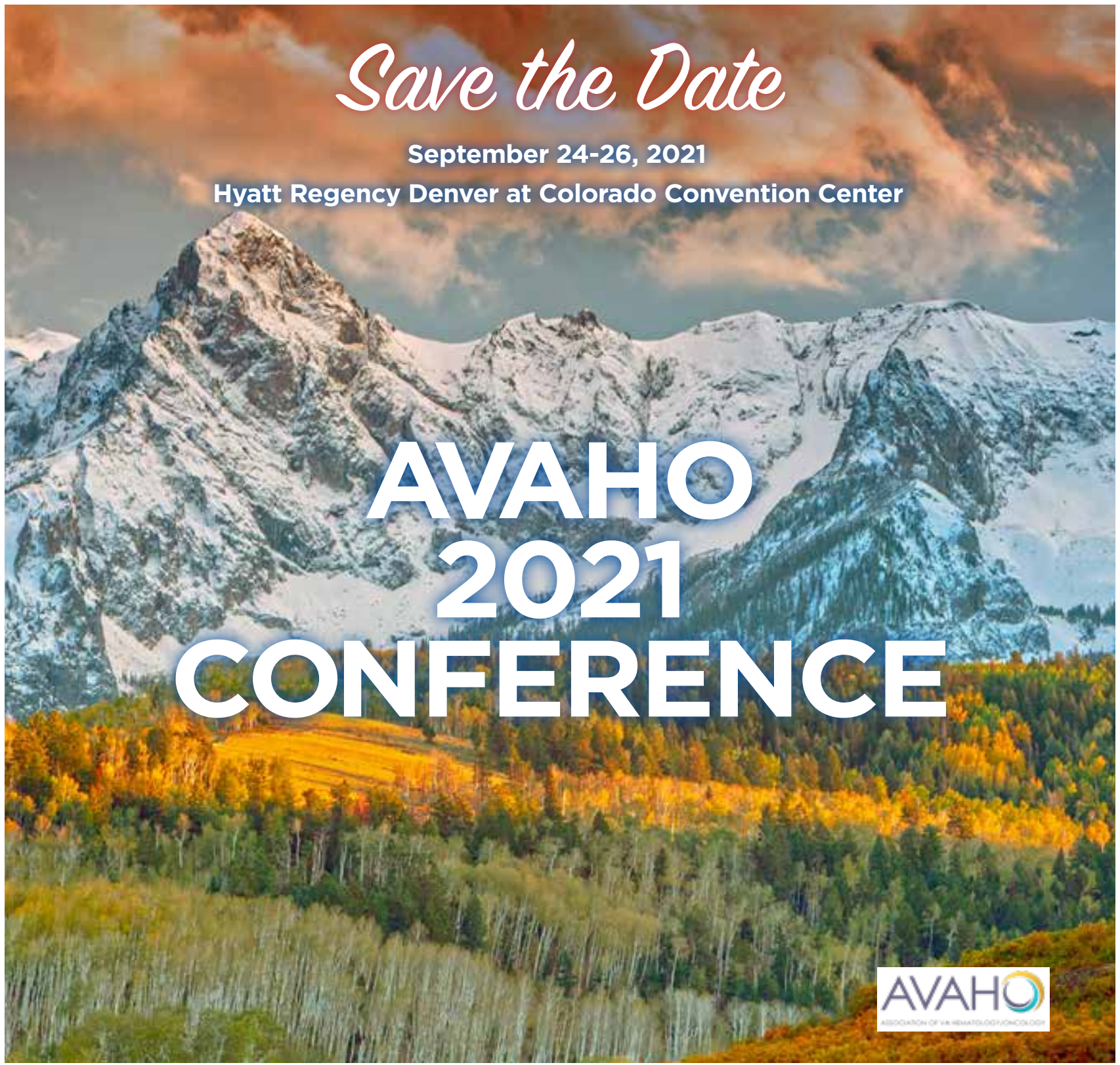

